\title{
Thyroid Hormone Receptor Agonist Promotes Hair Growth in Mice
}

\author{
Peiqiu Zhu ${ }^{1-5}$, Weiwei Deng ${ }^{1-5}$, Jin Yu ${ }^{1-5}$, Shuxia Yang ${ }^{1-5}$ \\ 'Department of Dermatology and Venereology, Peking University First Hospital, Beijing, People's Republic of China; ${ }^{2}$ Research Center for Medical \\ Mycology, Peking University, Beijing, People's Republic of China; ${ }^{3}$ Beijing Key Laboratory of Molecular Diagnosis on Dermatoses, Beijing, People's \\ Republic of China; ${ }^{4}$ National Clinical Research Center for Skin and Immune Diseases, Beijing, People's Republic of China; ${ }^{5}$ NMPA Key Laboratory for \\ Quality Control and Evaluation of Cosmetics, Beijing, People's Republic of China
}

Correspondence: Shuxia Yang; Jin Yu, Tel +86 83573103; +86 83573075, Email yangshuxia@bjmu.edu.cn; yujin676@I26.com

Background: Thyroxine is important to maintain the normal operation of the body. Both clinical and experimental results show thyroxine is closely related to hair growth, the mechanism of which is not fully understood.

Purpose: Investigate the effect of thyroxine receptor agonist, TDM10842, for dorsal hair growth in C3H mice and explore its underlying mechanism.

Methods: Depilated mice were applied with the TDM10842, vehicle of this drug and without any materials on dorsal skin. RNAsequencing (RNA-seq) was employed to identify the change in gene expression of skin tissues. Quantitative real-time PCR (rt-PCR) and immunoblotting were conducted to validate key differentially expressed genes (DEGs) between different groups.

Results: The TDM group showed early induction of anagen. 857, 782, and 276 differentially expressed genes were identified between 3 groups. As a critical DEG in group TDM, Pclaf was positively related to the motivation of Wnt/beta-catenin and Hedgehog signaling pathways, with a high expression of Ki67 and cyclinD1.

Conclusion: TDM10842 accelerates the anagen entrance and the potential mechanism might be the activation of Wnt/beta-catenin and Hedgehog pathways. Pclaf serves as a critical molecule involved in pathway activation, and cyclinD1 is an important effector protein downstream of the pathways.

Keywords: thyroid hormone receptor, $\mathrm{C} 3 \mathrm{H}$ mice, hair cycle, Pclaf, Wnt/beta-catenin pathway, Hedgehog pathway

\section{Introduction}

Thyroid hormone (TH) in adults is necessary for the regulation of multiple physiological effects, such as cell growth, structure, and metabolism. ${ }^{1}$ The main thyroid hormones include thyroxine (T4), 3,5,30-triiodothyronine (T3), and reverse 3,5,30-triiodothyronine (rT3). Controlled by thyroid-stimulating hormone (TSH), both T3 and T4 are secreted by the thyroid gland under physiological conditions. It is well known that thyroid dysfunction is associated with skin pathologies. Both abnormally low and excessively high serum levels of thyroid hormones (THs) can alter the appearance and function of human skin and its appendages, leading to pretibial myxedema and telogen effluvium. ${ }^{2}$ Hair follicle undergoes characteristic cyclic phases known as the hair cycle, including anagen, catagen, telogen, and exogen. It has been accepted that an intricated network of cellular and molecular signals involve in hair follicle morphogenesis and mediates these cycle transitions. Human HFs are direct targets of thyroid hormones and demonstrate that T3 and/or T4 modulate multiple hair biology parameters, ranging from HF cycling to pigmentation. ${ }^{3}$ Much of thyroid hormone action is mediated by the thyroid hormone receptors (TRs), members of the nuclear receptor superfamily that act as ligand-dependent transcription factors. ${ }^{4}$ Human skin and its appendages are known to express TH receptors on the gene and protein level, among which TR $ß 1$ is the predominant and functionally most relevant isoform. ${ }^{5}$ Whereas follicular proliferation and anagen entry is impaired in TRdeficient mice, with a reduced expression of Cyclin D1. ${ }^{6}$ Previous studies have shown that both T4 and the thyroid hormone analogue could prolong human hair growth in vitro, the latter of which decreased the expression of TGF-beta2. ${ }^{7}$ Whereas 
the underlying mechanism of how TR regulates and controls the hair cycle after activation still needs to be further elucidated, which could contribute to treatment strategies for hair loss diseases.

\section{Materials and Methods}

Ethics Statement

All experimental procedures and protocols used in this research were reviewed and approved by the animal care and use committee of the Peking University First Hospital. The institutional approval number for lab animal studies was 202036, with the Animal Management Regulations and Guidelines on The Good Treatment of Experimental Animals followed for the welfare of the laboratory animals.

\section{Experimental Animals and Drug Preparation}

Forty-five 8-week-old female $\mathrm{C} 3 \mathrm{H} / \mathrm{HeJ}$ mice were obtained in batches from Beijing Vital River Laboratory Animal Technology Co., Ltd., and then allowed to adapt for a week with food and water ad libitum. Mice were cared for in a controlled barrier facility with temperatures at $23 \pm 2{ }^{\circ} \mathrm{C}$, humidity at $35-60 \%$, and $12-\mathrm{h} \mathrm{light/dark}$ cycles. Mice were divided into three groups randomly: The Blank control group $(n=5)$, the Vehicle group $(n=5)$, and the TDM group $(\mathrm{n}=5)$. After confirming that all dorsal skin HF in telogen, as evidenced by the homogeneous pink skin color, ${ }^{8}$ depilation with VEET for 20 seconds, and then gently wipe the skin with sterile water immediately to remove the hair and cream. If not, the mouse would be excluded for further experimental steps. Wait 3 days to confirm there was no obvious skin damage resulted from the depilation procedure. As shown in Figure 1A, the depilated skin was the site with topical drug applied. The solvent consisted of 30\% propylene glycol and 70\% anhydrous ethanol. The TDM 10842, the agonist of thyroid hormone receptor, is a molecule compound synthesized and provided by TECHNODERMA MEDECINES PTE LTD, and prepared with a solvent at a concentration of $0.05 \%$. Exhibited in Figure 2A, which was the flow-chat conducted in this experiment, during every batch of operation, the Vehicle and TDM group were accepted twice application of solvents and drugs $(0,24 \mathrm{~h})$ with $60 \mathrm{ul}$ once a time and 3 mice of each teams sacrificed at $48 \mathrm{~h}$, remaining at least 1 mouse rested for observation (Figure 2A). The above experimental process was repeated independently 3 times, leading to total 36 mice for replicate experiments, 27 sacrificed for subsequent basic research and 9 for hair growth observation respectively. Anesthesia was applied whenever there was any procedure conducted on mice during depilation and medication.

\section{Histology}

Part of the skin tissues was fixed in $10 \%$ formalin immediately and dehydrated. The skin sample was transversely sectioned for paraffin embedding. The sections were cut longitudinally in paraffin (4-uM) and stained with hematoxylin and eosin (H\&E), then were examined under brightfield microscopy. Captures of digital images were counted in each sample, using NDP.view2 viewing software. The hair cycle staging was evaluated according to criteria by Sven MullerRover et al. ${ }^{8}$

\section{Histochemistry}

After fixed overnight in 10\% formalin, paraffin sections (4-uM) were deparaffinized and rehydrated. The slides were autoclaved in $10 \mathrm{mM}$ sodium citrate buffer for antigenic repairment. The sections were pre-incubated in PBS and then blocked at room temperature for $30 \mathrm{~min}$ with concentrated normal goat serum (Boster, AR1009). The sections were incubated with the following dilutions of primary antibodies: thyroid hormone receptor (TR) beta-1 (Thermofisher, 1:20), Ki-67 (Proteintech, 1:2000) overnight at $4^{\circ} \mathrm{C}$. The sections were rinsed with PBS and incubated with horseradish peroxidase and goat anti-mouse IgG secondary antibody (ZSGB, PV-9000) at room temperate, which were then reacted with DAB for coloration. The expression level of Ki67 was measured with ImageJ software. 
A

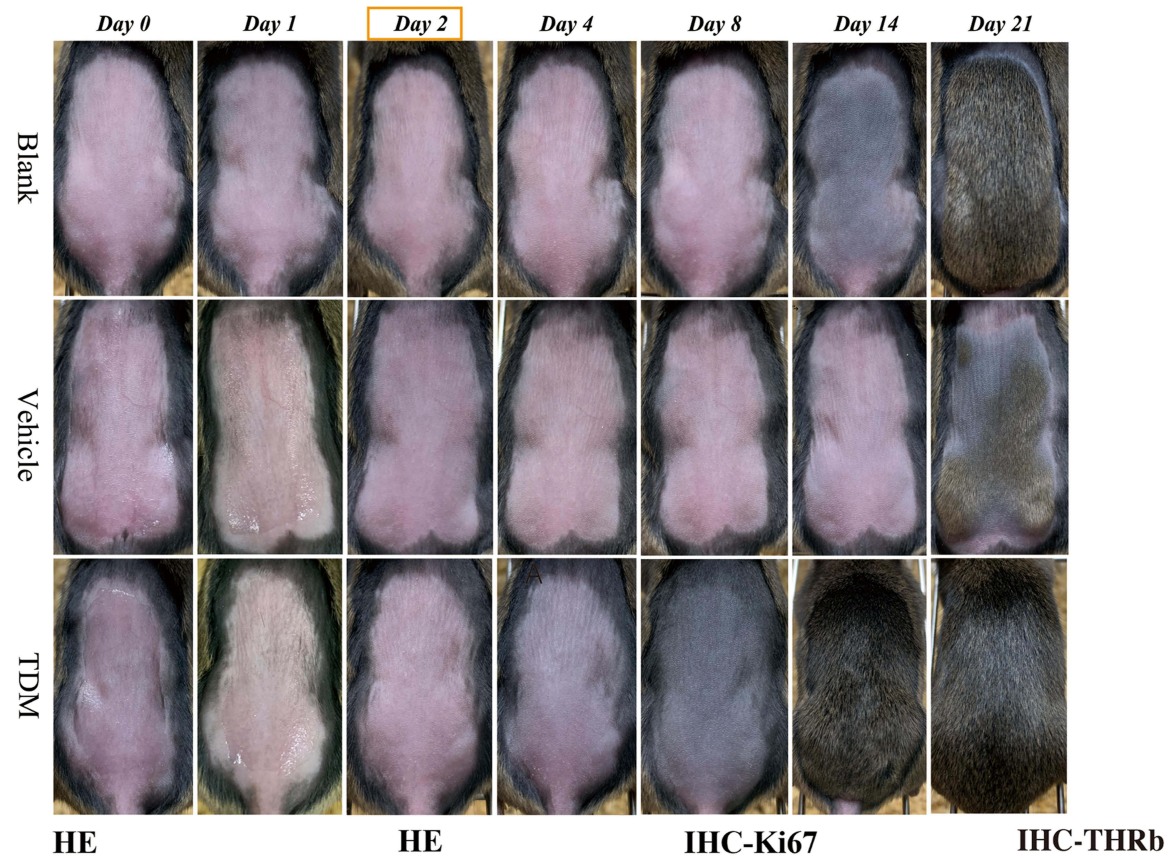

B

HE
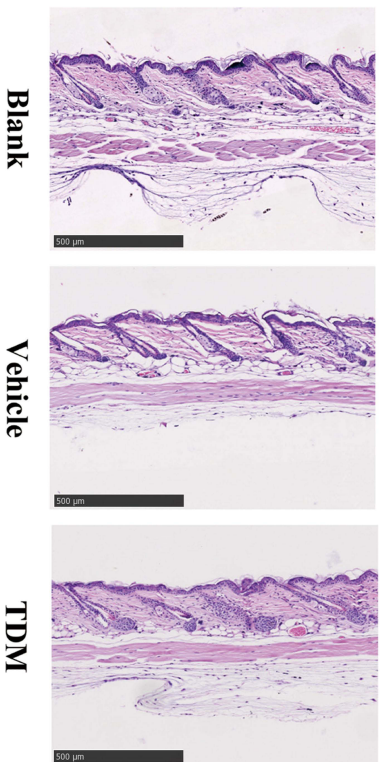

C
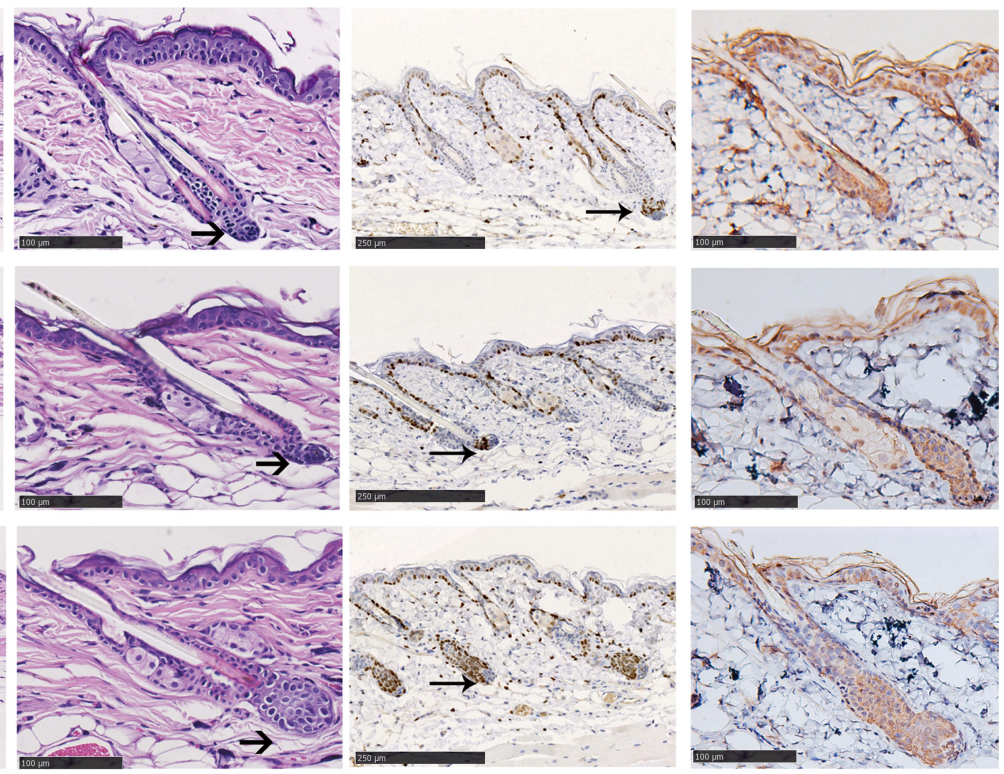

Hair cycle staging

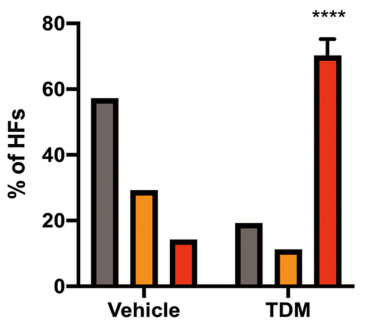

D

Proliferation

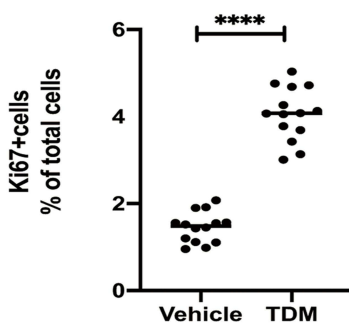

Figure I (A) Photographs of dorsal skins of C3H mice on the timepoint of Day 0, Day I, Day 2, Day 4, Day 8, Day I4, Day 2 I of the blank, vehicle and TDM treatment groups, respectively. The dorsal skin color of the TDM group turned grey on Day 4 and darker on Day 8 with hair shaft growing, compared with the other 2 groups. (B) H\&E, Thyroid hormone receptor beta-I and Ki-67 immunohistology images of the dorsal skin on the mice treated with blank control, vehicle control and TDMI0842 at the timepoint of Day 2. The hair bulb of the TDM group was enlarged than the other 2 groups and expanded into the subcutaneous tissue at the section from the H\&E perspective (as shown with the arrow). There was no significant difference of expression for thyroid hormone receptor beta-I between the 3 groups. The expression of Ki- 67 was increased in the follicular tissue of TDM group. (C) Results of microscopic hair cycle staging of two groups. Ratios of telogen, anagen I-II and anagen Illa hair follicles were calculated in each group. Data are mean standard error of the mean of the

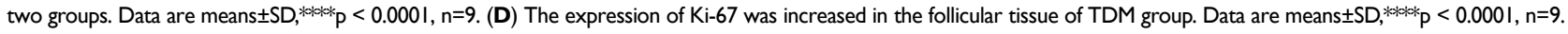



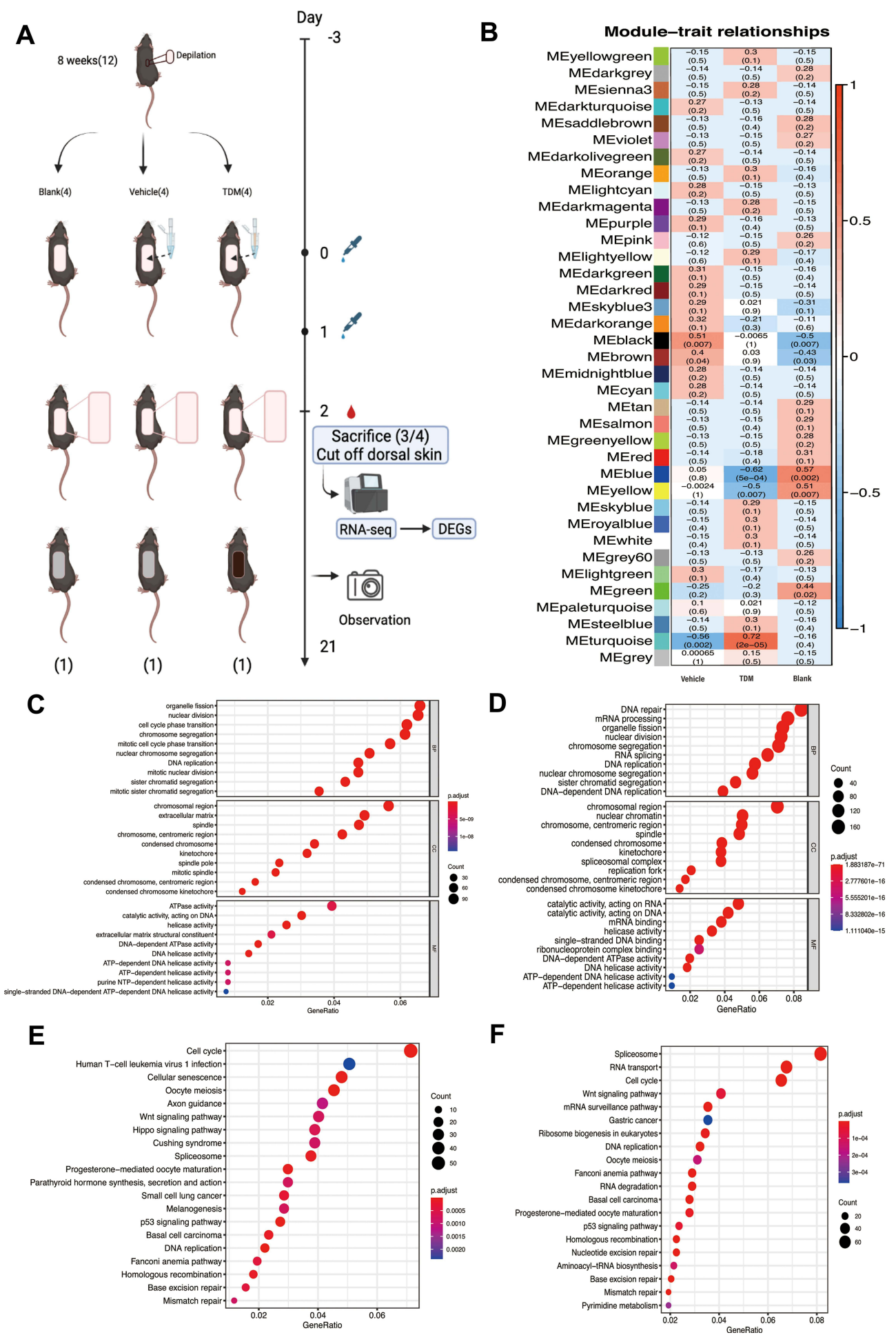

Figure 2 (A) The specific operation flow chart of the experiment. (B) The WGCNA analysis of the DEGs for 3 groups. The figure shows that the module most related to traits in the TDM group is pine green (MEturquoise). (C) The GO function analysis of the DEGs between TDM group and Vehicle group. The differences in biological processes are mainly manifested in organelle division, nuclear division, cell cycle transformation and so on. Cell composition are mostly focused on chromosome region, extracellular matrix, spindle, etc. Molecular functions are mainly involved in ATPase activity, catalytic enzyme activity acting on DNA, helicase activity, etc. (D) The GO function analysis of the DEGs between the TDM group and Blank group. The differences in biological processes are mainly reflected in DNA repair, mRNA processing, organelle division, etc. The difference in cell composition is reflected in the chromatin area, centromere area, spindle, etc. The difference in molecular function is mainly about the catalytic reaction activity acting on RNA, the catalytic reaction activity acting on DNA, mRNA binding, etc. (E) The KEGG analysis of the DEGs between TDM group and Vehicle group. Compared with the Vehicle group, the pathway enrichment of the TDM group is mainly reflected in the cell cycle, cell senescence, Wnt signaling pathway, etc. (F) The KEGG analysis of the DEGs between the TDM group and Blank group. Compared with the Blank group, the pathway enrichment of the TDM group is mainly manifested in differences in spliceosome, RNA transport, cell cycle, Wnt signaling pathway, and RNA monitoring pathway. 


\section{RNA Isolation and Complementary DNA Library Construction}

The TRIzol method (Invitrogen) was used to prepare total RNA from the dorsal skin tissue of $\mathrm{C} 3 \mathrm{H} / \mathrm{HeJ}$ mice. The purity and concentration of the RNA were measured using a NanoDrop 2000 spectrophotometer (ThermoFisher). The Agilent 2100 Bioanalyzer (Agilent) was used to detect RNA integrity number (RIN). Magnetic beads with poly-T oligo (Invitrogen) were applied to enrich mRNA, which was then fragmented with fragmentation buffer. Take the mRNA as template, complementary DNA (cDNA) was synthesized. Purified cDNA was afterwards processed to cohesive end repair, added with 3' base A. Selection was made after the cDNA was attached with the sequencing adapter based on fragment size. Finally, the cDNA library was obtained from PCR amplification. The constructed library was quality controlled using Agilent 2100 Bioanalyzer and ABI StepOnePlus Real-Time PCR System (ThermoFisher).

\section{RNA-Seq Data Analysis}

We gained the raw reads from the raw data of Illumina sequencing. The reads with cases of low quality, adaptor-polluted and the $\mathrm{N}$ bases number accounting for more than $5 \%$ were filtered out. The remaining data were filtered as clean reads. Match of the clean reads with the reference genome was conducted through HISAT. ${ }^{9}$ In order to calculate the gene alignment rate, we used Bowtie2 to complete the match of clean reads and the reference. ${ }^{10}$ RSEM was then used to calculate the expression levels of genes and transcripts. Raw counts were normalized by transcripts per million (TPM) values. DEGs were identified using $\mathrm{q} \leq 0.001$ and $\mid \log 2$ ratio $\mid \geq 1$. DEGs were functionally categorized by the GO database. Pathway enrichment of DEGs was implemented using the KEGG database. In order to screen out the genes with the most relevance for TDM treatment, WGCNA algorithm was employed. Cytoscape was used to visualize genes with the most relevance for TDM treatment. The detailed process was described in previous studies. ${ }^{11,12}$ The scores of pathways were identified using a recent pipeline. ${ }^{13}$ The relationships between genes and pathways were calculated using Pearson's correlation.

\section{Relative Quantification Real-time PCR (qPCR)}

The cDNAs were synthesized from RNA from reverse transcription of $1 \mu \mathrm{g}$ total RNA using a PrimeScript ${ }^{\mathrm{TMRT}}$ Reagent Kit with gDNA Eraser (Cat No. RR047A, TaKaRa) and oligo-dT primers in a total volume of $20 \mu \mathrm{L}$. Quantitative rt-PCR reactions were run in 20- $\mu \mathrm{L}$ reaction mixtures on an ABI Prism 7300 Real-Time PCR System (Applied Biosystems, CA, USA). PCR was performed with an initial preincubation step for $10 \mathrm{~min}$ at $95^{\circ} \mathrm{C}$, followed by 45 cycles of $95{ }^{\circ} \mathrm{C}$ for $15 \mathrm{~s}$, annealing at $52^{\circ} \mathrm{C}$ for $15 \mathrm{~s}$, and extension at $72{ }^{\circ} \mathrm{C}$ for $10 \mathrm{~s}$. The qPCR experiments were conducted in triplicate according to the instructions of the SYBR Green Premix AmpliTaq kit (Applied biosystems). The dissociation curve analysis was employed to confirm the amplification of a single product. Besides, primers for qPCR are given in Table S1. The qPCR $2-\Delta \Delta C t$ method was utilized to identify the relative mRNA expression of the selected hub genes. ${ }^{14}$

\section{Cell Culture and RNA Interference Experiments}

$\mathrm{HaCaT}$ cells were provided by the Chinese Academy of Sciences (Shanghai, China) and maintained in DMEM (Thermo Fisher, USA) supplemented with $10 \%$ fetal bovine serum (FBS; Thermo Fisher, USA) and $100 \mathrm{nM}$ penicillin/streptomycin (Life Technologies). 1*106/L cells were added in each well of 6-well plate with 3mL DMEM medium. The siRNA was synthesized by GenePharma as PCLAF-Homo-237 (5' to 3' S: GCCCAACUCCCAAGUGGCATT, AS: UGCCACUUGGGAGUUGGGCTT). Transfection was conducted according to Typical RNAiMAX Transfection Procedure (Lipofectamine 3000 Invitrogen). After $48 \mathrm{~h}$ of infection, cells were harvested and the knockdown of PCALF was verified by both immunoblotting and rt-PCR analysis.

\section{The Drug Experiment in vitro}

The TDM10842 was dissolved by dimethyl sulfoxide (DMSO) as mother liquid with a concentration of $10 \mathrm{mmol} / \mathrm{L}$. The mother liquid was then dissolved by Opti-MEM Medium and added into the medium to reach a final concentration of 5 umol/L in each well. The vehicle control was added with Opti-MEM Medium containing DMSO as the same concentration with the TDM group. We designed 4 groups, siRNA with DMSO, siRNA with TDM, Negative Control 
(NC) with DMSO, NC with TDM respectively. After 48h for co-incubation, cells were harvested for immunoblotting analysis.

\section{Immunoblotting}

RIPA (CST) was used to extract total protein from frozen tissues. BCA Protein Assay reagents (Thermo) were used to test total protein concentration. Total protein concentration was tested by BCA Protein Assay reagents (Beyotime Biotechnology). The protein electrophoresis was transferred to the PVDF membrane (Millipore) which was then incubated for first antibodies about 12 hours at $4{ }^{\circ} \mathrm{C}$. Then first antibodies were detected with secondary antibodies. Finally, the PVDF membrane was chemiluminescence with Tanon 5200 Multi Automatic Chemiluminescence system. Antibodies against to LEF1 (CST, 2230), Cyclin-D1 (CST, 55506S), PCLAF (CST, 81533), beta-Actin (Servicebio, GB12001).

\section{Statistical Analysis}

Statistical comparisons between groups were performed using two-tailed paired $t$-test analysis and/or Chi-square test by software (version 8.0, GraphPad). $\mathrm{P}<0.05$ was considered significant.

\section{Result}

\section{TDMI0842 Regulates the Hair Cycle and Promotes Hair Growth in vivo}

The progression of hair follicle development in mice was evaluated by images captured on the first day of application of medicine and 1,2-, 4-, 8, 14- and 28-days following medication. The skin color turned greyish on the mice dorsal at 4 days post-medication compared with the 2 control groups, especially at 8 days with the emergence of the hair shaft (Figure 1A), the in-vitro result was same in every batch of operation. To further assess the size of hair follicles, skin biopsy sections were obtained from the mice and stained with H\&E. The skin biopsy confirmed that the size of hair follicles was markedly increased in TDM group compared to the two control groups (Figure 1B). The proportion of hair follicles in the anagen phase of experimental group was significantly higher than that in the control group (Figure 1C). The expression of Ki67, indicating proliferation index, was significantly increased in the hair follicles of the drug group (Figure 1B-D). Immunohistochemical results of thyroxine receptor confirmed that there was no significant difference in receptor expression among the three groups (Figure 1B).

\section{Analysis of WGCNA, GO, and KEGG Pathways}

We found 857, 782, and 276 differentially expressed genes were identified from the skin tissue between 3 groups, respectively (NCBI database,GSE192797). To identify the function of DEGs, we employed Gene Ontology (GO) function analyses using the GO database, Kyoto Encyclopedia of Genes and Genomes (KEGG) function analyses using the KEGG database. As shown in Figure 2C and D, the results of GO classification manifested that there were several enriched GO terms in the TDM group, which mainly involve in organelle fission, nuclear division, cell cycle phase transition compared with the Vehicle group, and DNA repair, mRNA processing, organelle fission compared with the Blank group, respectively. As can be seen in Figure 2E and F, compared with the other groups, the pathway enrichment of the TDM group were dominantly focused on the cell cycle and Wnt signaling pathway. Weighted correlation network analysis (WGCNA) was used to find clusters of highly correlated genes from the 3 groups. As Figure 2B shows, the module of METurquoise was extremely relevant with the TDM group. From which, we found one gene, Pclaf, as a differentially expressed gene compared with the other two groups (Figure S1). Importantly, Pclaf has been reported involved in the Wnt signaling pathway. ${ }^{15}$

\section{Activated Pathway Score and Correlation Between Key Differentially Expressed Genes with Hedgehog Signaling and Wnt/Beta-Catenin Signaling Pathway}

To further characterize the heterogeneity of pathways between different treatments, we scored their pathway activities. We found that signaling pathway of E2F TARGETS, G2M CHECKPOINT, MYC TARGET V1, HEDGEHOG 
A

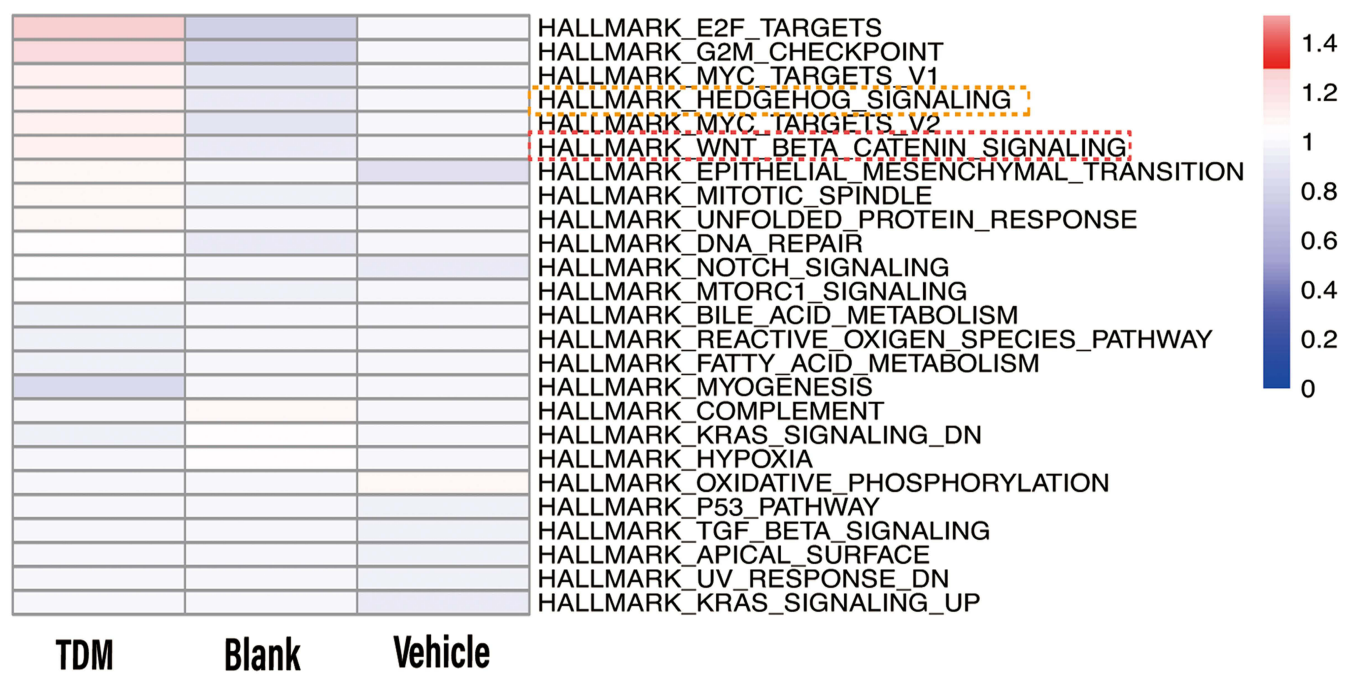

B

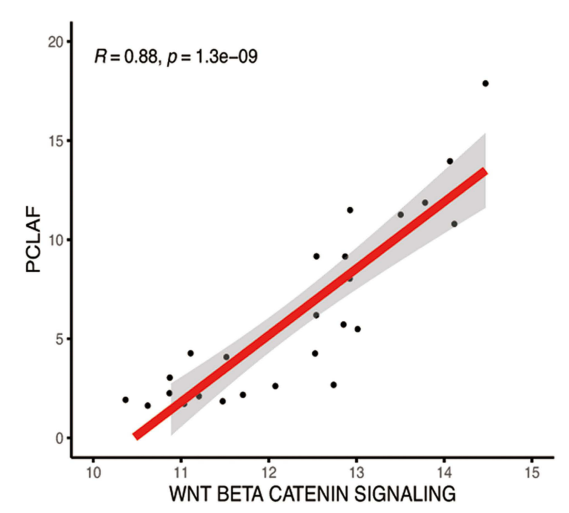

1.4

1.2

0.8

0.6

0.4

0.2
C

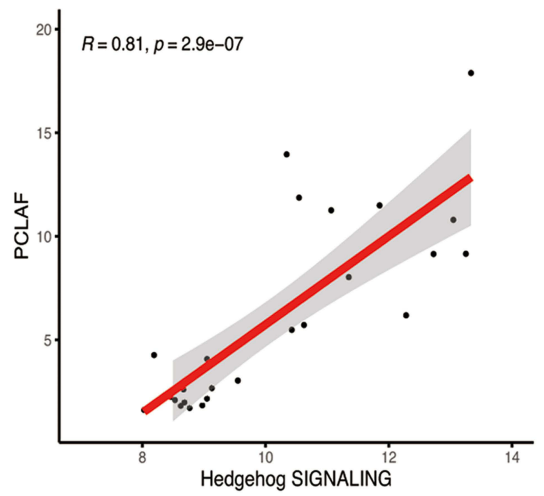

D

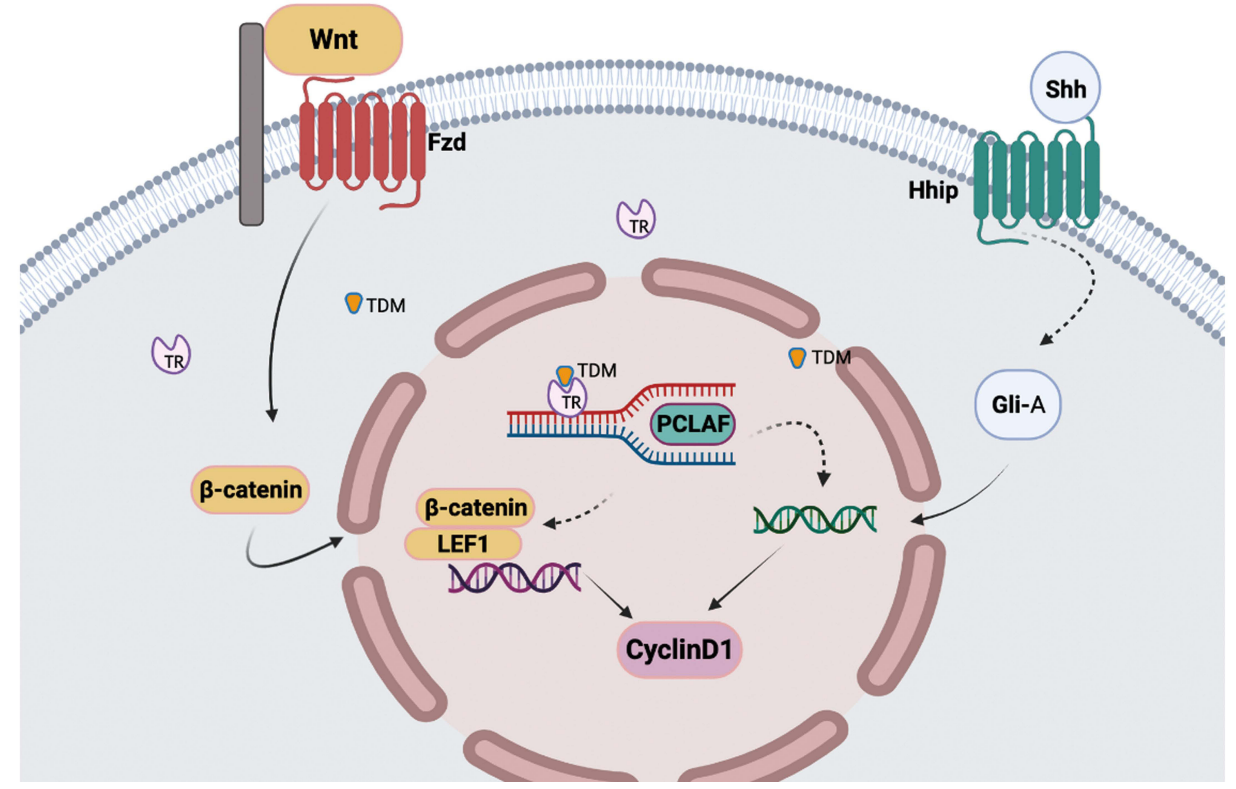

Figure 3 (A) The activated level of different pathways between the 3 groups. (B) The Pearson relationship between Pclaf and the Wnt signaling pathway is 0.88 . (C) The Pearson relationship between Pclaf and the Hedgehog signaling pathway is 0.81 . (D) The main up-regulated genes analyzed by RNA-seq data were labeled and drew by Bio render. 
SIGNALING, MYC TARGET V2, WNT BETA CATENIN SIGNALING, EPITHELIAL MESENCHYMAL TRANSITION, MITOTIC SPINDLE, UNFOLDED PROTEIN RESPONSE and DNA REPAIR were up regulated in the TDM group compared with the other 2 control groups (Figure 3A). Then, we explored the potential relationship between Pclaf and WNT SIGNALING PATHWAY, as well as with HEDGEHOG SIGNALING PATHWAY, which show strongly positive correlations ( $\mathrm{R}=0.88$ and 0.81 , respectively) (Figure $3 \mathrm{~B}$ and $\mathrm{C}$ ). The main up-regulated genes in Wnt/ beta-catenin and Shh pathways analyzed by RNA-seq data were labeled and drew by Bio render (Figure 3D).

\section{Relative Quantification Real-time PCR of Pclaf and Other DEGs in Hedgehog and Wnt/Beta-Catenin Signaling Pathway and Validation of Key DEGs by Western Blotting in vivo}

Besides Pclaf, we selected 8 genes from the Wnt/beta catenin signaling pathway and 6 genes from the HedgeHog signaling pathway to validate the gene expression (Figure 4A and B). Selected genes played important roles in the pathway. The mRNA level of Pclaf, Wnt5a, Wnt5b, Fzd1, Fzd2, Ctnnb1, Lef1, Ccnd1, Shh, Hhip, Gli1, Gli2, Ptch1 and, Ptch2 were significantly increased in group of TDM. Altogether, these results revealed that Wnt/beta-catenin and HedgeHog signaling pathways may play crucial roles in hair growth after TDM treatments.

\section{TDMI0842 Promotes Expression of PCLAF in HaCat Cells, Silencing of PCLAF Down-Regulates Downstream Proteins Expression of Cyclin-DI}

To further investigate the role of PCLAF on Wnt/beta-catenin and Hedgehog signaling pathways, we utilized the small interfering RNA (siRNA) targeting the expression of PCLAF to transfected human HaCat cells. The knockdown efficiency was confirmed by rt-PCR and western blotting (Figure 4D and E). Compared to the negative control, we found that silencing of PCLAF significantly reduced the expression of LEF1 and Cyclin-D1 (Figure 4E). Furthermore, TDM10842 could significantly enhanced the expression of PCLAF under the condition of whether PCLAF was transfected or not, along with LEF1 and Cyclin-D1 (Figure 4F).

\section{Discussion}

Previous studies suggest that thyroid hormone significantly affect hair growth, TRs definitely play a critical role in this biological process. ${ }^{16,17}$ Herein we want to further explore the potential molecular mechanism of the hair cycle phase transition after receptor activation. In terms of experimental design, we made use of a $\mathrm{C} 3 \mathrm{H}$ mice model based on the wavy growth pattern of the back hair. ${ }^{8}$ Shave with clippers were used during preliminary experiments, whereas punctate areas with hair growth were observed. Aim to investigate the potential changes from transcriptomics after TR receptor activation, we set up 3 groups, with VEET depilation as the unified background, and gave different stimulus to explore differences between the TDM and Vehicle groups. We found TDM10842 could accelerate the murine hair cycle transition from telogen to anagen. Besides, the weight gain was stable and with normal behavior observed between 3 groups. On the basis of this appearance, the potential underlying causes of an earlier anagen phase entry was further explored from outside to inside.

Canonical TH signaling is based on the interaction with nuclear TRs, leading to either activation or repression of the transcription of a large number of genes. ${ }^{18}$ In addition to canonical signaling, TH may activate a number of noncanonical signaling pathways. ${ }^{19}$ The latter involves TRs that do not bind DNA directly, extranuclear TRs that activate the phosphatidylinositol 3-kinase pathway, and membrane receptors belonging to the integrin family. ${ }^{20}$ With regard to our research, the possible mechanism of hair growth cycle regulation by TR agonists is complex and should include both intra- and extracellular TR activation (Figure 3D). RNA-seq data suggests that TDM10842 promotes both enrichment of extracellular matrix and chromosomal regions and upregulation of biological process related to organelle fission, nuclear division and cell cycle phase transition. In addition, we identify a novel role of Pclaf within TR-related anagen induction, especially the activation of Wnt/beta-catenin and sonic hedgehog (Shh) signaling pathways, which were definitely related to hair follicle organogenesis and cycling controlling. ${ }^{21-24}$ 
A
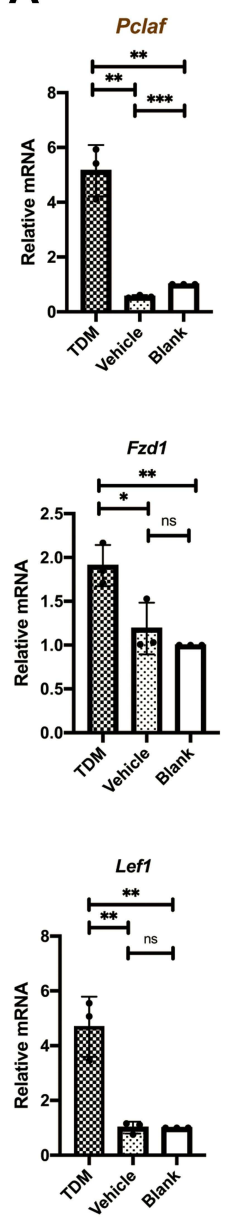
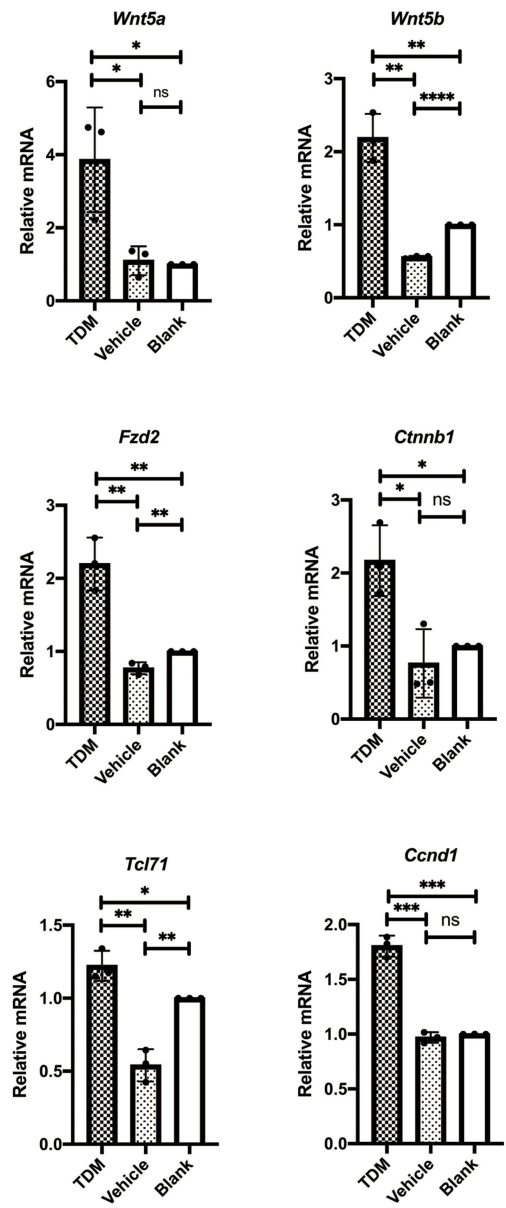

B
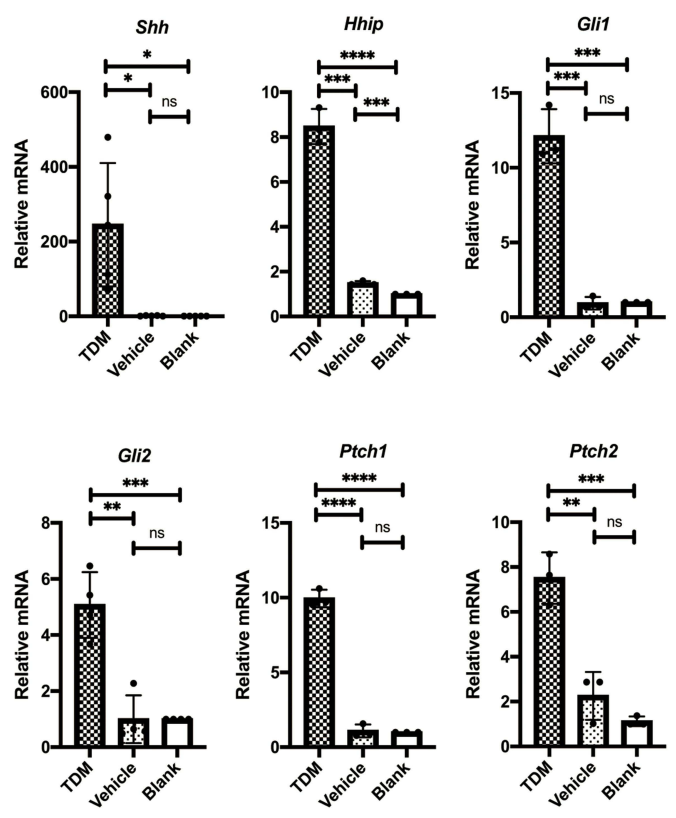

C

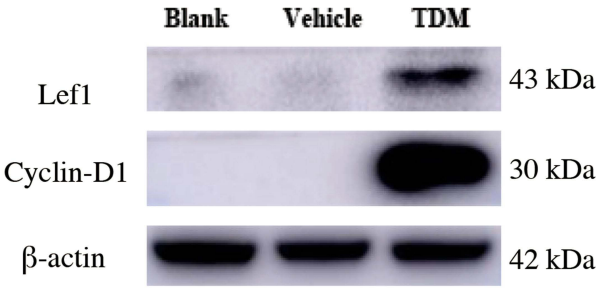

F

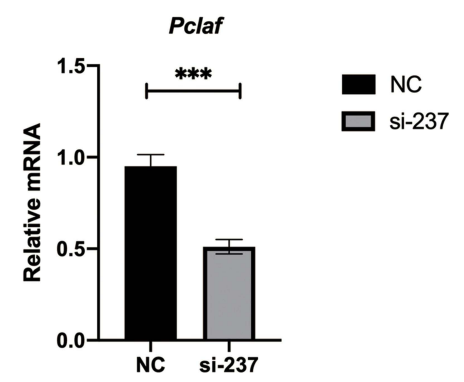

$\mathbf{E}$

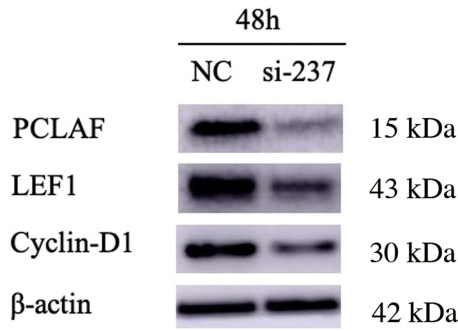

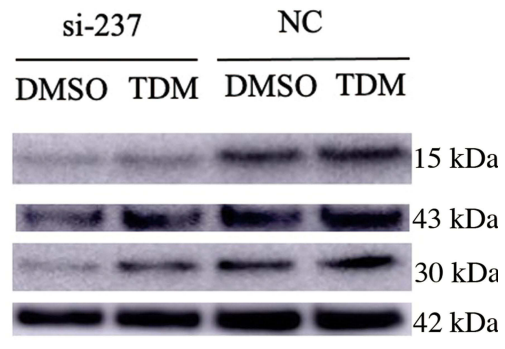

Figure 4 (A) Compared with the other 2 groups, the expression of Pclaf increased significantly. Genes related to the Wnt pathway: Wnt5a, Wnt5b, FzdI, Fzd2, CtnnbI, LefI, Tcl7I, and CcndI all showed different degrees of increase in expression. Data are means \pm SD, ${ }^{*} p<0.05$, ${ }^{* *} p<0.01, * * * p<0.00 I, * * * * p<0.000 \mathrm{I}, \mathrm{n} \geq 3$. (B) Shh, Hhip, Glil, Gli2, PtchI, Ptch2, as the Hedgehog signaling pathway-related genes, were significantly highly expressed in the TDM group. Data are means \pm SD, *p< 0.05 , $* * p<0.01$, $*_{* *} \mathrm{p}<0.00 \mathrm{I}, * * * * \mathrm{p}<0.000 \mathrm{I}, \mathrm{n} \geq 3$. (C) The protein expression of Lefl and CyclinDI in the TDM group was higher than that in the 2 control groups. (D) Knockdown efficiency of Pclaf after $48 \mathrm{~h}$ in HaCat cells confirmed by rtPCR. Data are means \pm SD, ${ }^{*} p<0.05, * * p<0.01$, $* * * p<0.001, n=3$. (E) The knockdown efficiency of Pclaf after $48 \mathrm{~h}$ in $\mathrm{HaCat}$ cells confirmed by immunoblotting. (F) Knocking down of PCLAF in HaCat cells with TDMI 0842 co-incubated $48 \mathrm{~h}$ showed slightly high expression of PCLAF, LEFI and Cyclin-DI compared with DMSO. Without siRNA targeted PCALF, TDMI0842 promoted PCLAF, LEFI and Cyclin-DI expression in HaCat cells compared with DMSO after $48 \mathrm{~h}$.

We observed that earlier anagen entrance inducted by TR activation mainly motivated Wnt- and Shh signaling, whereas TR-beta KO follicle stem cells reduced activation resulted from aberrant activation of Smad signaling. ${ }^{25}$ Smad proteins were phosphorylated by bone morphogenetic proteins (BMPs). ${ }^{26}$ Intranuclear P-Smad together with Smadinteracting proteins and target genes is present in the resting bulge, indicating a role for Smad-BMP signals in 
maintaining quiescence of hair follicle SCs. Our study indicated that the Smad-BMP signaling was not involved in hair cycle regulating of TR activation. Wnt signaling is indeed regarded as one of the most fundamental pathways for anageninduction, driving murine anagen-induction also occurring in human scalp HFs. ${ }^{23}$ However, Wnt7b, considered as an important intrinsic regulator of hair follicle stem cell homeostasis and hair cycling, ${ }^{27}$ was not observed stimulating in our research neither in RNA-seq nor in transcriptional level. Different from the thyroid hormone analogue, ${ }^{7}$ which was verified to promote human hair growth by reduced the expression of TGF-beta, we found the TR agonist was obviously focused on Wnt-beta catenin and its target gene cyclin D1 to promote murine hair growth. Our studies also showed that hyperactivation of the Shh signaling pathway at anagen onset has resulted from TR activation. Shh is crucial for hair development and cycling. ${ }^{28} \mathrm{Hh}$-agonist has been shown to stimulate the transition from the resting (telogen) to the growth (anagen) stage of the hair cycle in adult mouse skin. ${ }^{29}$ Further studies indicated that Hes1, a major Notch downstream transcriptional repressor, reinforces the Shh signaling during the telogen-anagen transition to maintain hair cycle homeostasis. $^{30}$

Interesting, the crosstalk between Wnt- and Shh- signals not only exists in the hair-generating process but also in the hair cycle transition. Our research showed that this point of interaction is both reflected in the expression of Pclaf and the high expression of the downstream target gene CyclinD1 (Figures 3 and 4). Notably, we found Pclaf was not only the most associated with TDM group module traits but also a significantly DEG in the TDM group. A previous study revealed that PCLAF (PCNA clamp associated factor), played an unexpectedly pivotal role in the enhancement of transcription of Wnt target genes. ${ }^{15}$ As a DNA repair gene, Pclaf was shown to be involved in translation DNA synthesis (TLS), an error-prone DNA-repair process that permits DNA-replication machinery to replicate DNA lesions with specialized translation DNA polymerase, its overexpression may play a dual role in inducing cell hyperproliferation in cancer. ${ }^{31}$ However, there is no evidence that Pclaf is related to anagen induction of hair follicles regulated by the Wntsignaling or Shh- pathways or the connection between TR and Pclaf. We first revealed that Pclaf may correlated with the activating Wnt- and Shh- pathways in TR promoting hair growth model, which draws a new thread for molecular mechanism research underlying the telogen-anagen transition of murine hair follicle cycle (Figure 3). Regrettably, we cannot get access to murine species reactivity antibody for Pclaf, so the immunochemistry and protein experimental validation from the tissue level were unable to proceed. Meanwhile, our results suggest that TDM10842 promoted expression of PCLAF and LEF1 and Cyclin-D1 were positively regulated by PCLAF. There were no studies focused on the correlation between thyroid hormone receptors and PCLAF currently. We hypothesize if Pclaf is one of the thyroid hormone response elements to regulate target genes or bind receptor molecules. Although we proved that the activation of thyroid hormone receptor could promote PCLAF related downstream protein expression within Wnt-beta catenin and Shh signaling pathways. The upstream signals resulting in Pclaf activating the LEF1 and Cyclin-D1 remains unclear and requires further investigation.

Cyclin D1 acts as a mitogenic sensor and integrates excellular and mitogenic signals and cell cycle progression, to control the progression through the G1 phase, the G1-to-S phase transition, thereby initiating DNA replication. ${ }^{32}$ During hair follicle organogenesis, cyclin D1 expression is induced by Shh- or Wnt10b-signaling and the role of Shh in its expression is indispensable. ${ }^{33}$ Previous studies have shown that the natural thyroid hormone analogue could stimulate epidermal thickening in mice and promote human keratinocyte proliferation by activating Cyclin D1 expression and promoting entrance into the S phase. ${ }^{34}$ Our studies indicated that thyroid receptor agonists could accelerate anagen entrance of hair follicles through motivating Cyclin D1 expression. More in-depth research could focus on whether and how cyclinD1 promotes the specific mechanism of hair follicle stem cells to maintain stemness and promote cell division. TDM10842 may affect the self-renewal, cycle regulation, and cell division of hair follicle stem cells and other precursor cells of the skin, which may provide new thoughts for the treatment of hair loss populations (androgenetic alopecia, alopecia areata and telogen effluvium, etc.).

Although we found an evident difference in hair cycle transition between control and TDM groups, as well as identifying Pclaf involved in regulating Wnt and Shh pathways and the possible downstream transcriptional targets, the upstream process resulting in activation and translation remain unclear and deserve further investigation. In addition, hair follicle stem cells or dermal papilla cells could better verify the function of cell cycle change and hair follicle growth of 
TDM10842. Hence, further drug stimulation at the dermal papilla level is being considered, so as to explore how TDM10842 promotes the anagen entrance of hair follicles and whether it can prolong the anagen period.

\section{Conclusion}

In conclusion, here we have confirmed the effect of thyroid hormone receptor agonists on the hair cycle and revealed its latent mechanism through in vivo experiments, transcriptome analysis and successive verification. The preliminary experimental results show that topical thyroid hormone receptor agonists can promote hair follicles in telogen to enter the anagen. RNA-sequencing is used to screen the DEGs between different treatment groups. The results of signaling pathways activation scoring and level verification show that the mechanism is related to the upregulation of Wnt/betacatenin and Hedgehog signaling pathways. Pclaf may be an important molecule involved in pathway activation, and cyclinD1 is an important effector protein downstream of the pathways.

\section{Disclosure}

The authors report no conflicts of interest in this work.

\section{References}

1. Chi HC, Chen CY, Tsai MM, et al. Molecular functions of thyroid hormones and their clinical significance in liver-related diseases. Biomed Res Int. 2013;2013:601361. doi:10.1155/2013/601361

2. Paus R. Exploring the "thyroid-skin connection": concepts, questions, and clinical relevance. J Invest Dermatol. 2010;130(1):7-10. doi:10.1038/ jid.2009.359

3. van Beek N, Bodo E, Kromminga A, et al. Thyroid hormones directly alter human hair follicle functions: anagen prolongation and stimulation of both hair matrix keratinocyte proliferation and hair pigmentation. J Clin Endocrinol Metab. 2008;93(11):4381-4388. doi:10.1210/jc.2008-0283

4. Anyetei-Anum CS, Roggero VR, Allison LA. Thyroid hormone receptor localization in target tissues. J Endocrinol. 2018;237(1):R19-R34. doi:10.1530/JOE-17-0708

5. Billoni N, Buan B, Gautier B, et al. Thyroid hormone receptor beta1 is expressed in the human hair follicle. Br J Dermatol. 2000;142(4):645-652. doi:10.1046/j.1365-2133.2000.03408.x

6. Contreras-Jurado C, Garcia-Serrano L, Martinez-Fernandez M, et al. Impaired hair growth and wound healing in mice lacking thyroid hormone receptors. PLoS One. 2014;9(9):e108137. doi:10.1371/journal.pone.0108137

7. Olah A, Gherardini J, Bertolini M, et al. The thyroid hormone analogue KB2115 (eprotirome) prolongs human hair growth (anagen) ex vivo. $J$ Invest Dermatol. 2016;136(8):1711-1714. doi:10.1016/j.jid.2016.03.033

8. Muller-Rover S, Handjiski B, van der Veen C, et al. A comprehensive guide for the accurate classification of murine hair follicles in distinct hair cycle stages. $J$ Invest Dermatol. 2001;117(1):3-15. doi:10.1046/j.0022-202x.2001.01377.x

9. Kim D, Langmead B, Salzberg SL. HISAT: a fast spliced aligner with low memory requirements. Nat Methods. 2015;12(4):357-360. doi:10.1038/ nmeth.3317

10. Langdon WB. Performance of genetic programming optimised bowtie2 on genome comparison and analytic testing (GCAT) benchmarks. Bio Data Min. 2015;8(1):1.

11. Deng W, Su Z, Liang P, et al. Single-cell immune checkpoint landscape of PBMCs stimulated with candida albicans. Emerg Microbes Infect. 2021;10(1):1272-1283. doi:10.1080/22221751.2021.1942228

12. Langfelder P, Horvath S. WGCNA: an R package for weighted correlation network analysis. BMC Bioinform. 2008;9:559. doi:10.1186/1471-21059-559

13. Xiao Z, Dai Z, Locasale JW. Metabolic landscape of the tumor microenvironment at single cell resolution. Nat Commun. 2019;10(1):3763. doi:10.1038/s41467-019-11738-0

14. Livak KJ, Schmittgen TD. Analysis of relative gene expression data using real-time quantitative PCR and the 2 (-delta delta C (T)) method. Methods. 2001;25(4):402-408. doi:10.1006/meth.2001.1262

15. Jung HY, Jun S, Lee M, et al. PAF and EZH2 induce Wnt/beta-catenin signaling hyperactivation. Mol Cell. 2013;52(2):193-205. doi:10.1016/j. molcel.2013.08.028

16. Lause M, Kamboj A, Fernandez Faith E. Dermatologic manifestations of endocrine disorders. Transl Pediatr. 2017;6(4):300-312. doi:10.21037/ tp.2017.09.08

17. Pascual A, Aranda A. Thyroid hormone receptors, cell growth and differentiation. Biochim Biophys Acta. 2013;1830(7):3908-3916. doi:10.1016/j. bbagen.2012.03.012

18. Zucchi R. Thyroid hormone analogues: an update. Thyroid. 2020;30(8):1099-1105. doi:10.1089/thy.2020.0071

19. Flamant F, Cheng SY, Hollenberg AN, et al. Thyroid hormone signaling pathways: time for a more precise nomenclature. Endocrinology. 2017;158 (7):2052-2057. doi:10.1210/en.2017-00250

20. Davis PJ, Mousa SA, Lin HY. Nongenomic actions of thyroid hormone: the integrin component. Physiol Rev. 2021;101(1):319-352. doi:10.1152/ physrev.00038.2019

21. Stenn KS, Paus R. Controls of hair follicle cycling. Physiol Rev. 2001;81(1):449-494. doi:10.1152/physrev.2001.81.1.449

22. Qiu W, Lei M, Zhou L, et al. Hair follicle stem cell proliferation, Akt and Wnt signaling activation in TPA-induced hair regeneration. Histochem Cell Biol. 2017;147(6):749-758. doi:10.1007/s00418-017-1540-1

23. Hawkshaw NJ, Hardman JA, Alam M, et al. Deciphering the molecular morphology of the human hair cycle: wnt signalling during the telogenanagen transformation. Br J Dermatol. 2020;182(5):1184-1193. doi:10.1111/bjd.18356 
24. Lim CH, Sun Q, Ratti K, et al. Hedgehog stimulates hair follicle neogenesis by creating inductive dermis during murine skin wound healing. Nat Commun. 2018;9(1):4903. doi:10.1038/s41467-018-07142-9

25. Contreras-Jurado C, Lorz C, Garcia-Serrano L, et al. Thyroid hormone signaling controls hair follicle stem cell function. Mol Biol Cell. 2015;26 (7):1263-1272. doi:10.1091/mbc.E14-07-1251

26. Fuchs E, Chen T. A matter of life and death: self-renewal in stem cells. EMBO Rep. 2013;14(1):39-48. doi:10.1038/embor.2012.197

27. Kandyba E, Kobielak K. Wnt7b is an important intrinsic regulator of hair follicle stem cell homeostasis and hair follicle cycling. Stem Cells. 2014;32(4):886-901. doi:10.1002/stem.1599

28. Rishikaysh P, Dev K, Diaz D, et al. Signaling involved in hair follicle morphogenesis and development. Int J Mol Sci. 2014;15(1):1647-1670. doi:10.3390/ijms 15011647

29. Paladini RD, Saleh J, Qian C, et al. Modulation of hair growth with small molecule agonists of the hedgehog signaling pathway. J Invest Dermatol. 2005;125(4):638-646. doi:10.1111/j.0022-202X.2005.23867.x

30. Suen WJ, Li ST, Yang LT. Hes1 regulates anagen initiation and hair follicle regeneration through modulation of hedgehog signaling. Stem Cells. 2020;38(2):301-314. doi:10.1002/stem.3117

31. Povlsen LK, Beli P, Wagner SA, et al. Systems-wide analysis of ubiquitylation dynamics reveals a key role for PAF15 ubiquitylation in DNAdamage bypass. Nat Cell Biol. 2012;14(10):1089-1098. doi:10.1038/ncb2579

32. Tchakarska G, Sola B. The double dealing of cyclin D1. Cell Cycle. 2020;19(2):163-178. doi:10.1080/15384101.2019.1706903

33. Schmidt-Ullrich R, Tobin DJ, Lenhard D, et al. NF-kappaB transmits Eda A1/EdaR signalling to activate Shh and cyclin D1 expression, and controls post-initiation hair placode down growth. Development. 2006;133(6):1045-1057. doi:10.1242/dev.02278

34. Zhang B, Zhang A, Zhou X, et al. Thyroid hormone analogue stimulates keratinocyte proliferation but inhibits cell differentiation in epidermis. Int J Immunopathol Pharmacol. 2012;25(4):859-869. doi:10.1177/039463201202500404

Clinical, Cosmetic and Investigational Dermatology is an international, peer-reviewed, open access, online journal that focuses on the latest clinical and experimental research in all aspects of skin disease and cosmetic interventions. This journal is indexed on CAS. The manuscript management system is completely online and includes a very quick and fair peer-review system, which is all easy to use. Visit http://www. dovepress.com/testimonials.php to read real quotes from published authors.

Submit your manuscript here: https://www.dovepress.com/clinical-cosmetic-and-investigational-dermatology-journal 